\title{
Modified FOLFOX6 as a first-line treatment for patients with advanced gastric cancer with massive ascites or inadequate oral intake
}

This article was published in the following Dove Press journal: OncoTargets and Therapy

\section{Hiroki Osumi \\ Daisuke Takahari \\ Keisho Chin \\ Mariko Ogura \\ Takashi Ichimura \\ Takeru Wakatsuki \\ Takeshi Suzuki \\ Yumiko Ota \\ Izuma Nakayama \\ Akira Ooki \\ Mitsukuni Suenaga \\ Eiji Shinozaki \\ Kensei Yamaguchi}

Department of Gastroenterology, Cancer Institute Hospital, Japanese Foundation for Cancer Research,

Tokyo, Japan
Correspondence: Daisuke Takahari Department of Gastroenterology, Cancer Institute Hospital, Japanese Foundation for Cancer Research, 3-8-3I Ariake, Koto-ku, Tokyo I35-8550, Japan

$\mathrm{Tel}+8 \mathrm{I} 3352001 \mathrm{II}$

Fax +81335700343

Email daisuke.takahari@jfcr.or.jp
Background: Oral fluoropyrimidine plus platinum is a standard first-line treatment for advanced gastric cancer (AGC). However, this treatment is problematic for AGC patients with massive ascites or inadequate oral intake. This study aimed at evaluating the efficacy and safety of modified oxaliplatin (L-OHP) with 1-leucovorin (1-LV) and bolus/continuous infusion of 5-fluorouracil (5-FU) (mFOLFOX6) regimen for patients with massive ascites or inadequate oral intake.

Methods: This retrospective study was conducted at a single Japanese institute from November 2015 to May 2018. The mFOLFOX6 regimen consisted of $85 \mathrm{mg} / \mathrm{m}^{2} \mathrm{~L}-\mathrm{OHP}, 400 \mathrm{mg} / \mathrm{m}^{2}$ bolus of 5-FU, and $400 \mathrm{mg} / \mathrm{m}^{2} 1$-LV on the first day, followed by $2,400 \mathrm{mg} / \mathrm{m}^{2}$ of 5 -FU as a continuous infusion in 46 hours for first-line treatment. The definition of inadequate oral intake was the need for total parenteral nutrition (TPN). Massive ascites was defined as continuous ascites from the pelvic cavity to the upper abdomen. Improvement in oral intake was defined as no TPN for more than 7 days, and improvement in ascites was defined as a decrease in ascites of more than one grade defined by the Japan Clinical Oncology Study Group trial (JCOG0106).

Results: Among the 364 patients with AGC who received first-line chemotherapy, 17 patients (13 [76.5\%] had inadequate oral intake, and four [23.5\%] had massive ascites) were enrolled in this study. Median time to treatment failure and overall survival were $4.8(95 \% \mathrm{CI}=1.5-7.5)$ and 8.8 months $(95 \% \mathrm{CI}=2.3-$ not available), respectively. Objective improvements in oral intake and ascites were seen in 11 of 13 patients $(84.6 \%)$ and 6 of 12 patients $(50 \%)$, respectively. The major grade 3 or 4 adverse events were neutropenia (35.3\%), febrile neutropenia (5.9\%), fatigue $(5.9 \%)$, anorexia (5.9\%), and infection (5.9\%). No treatment-related deaths occurred.

Conclusion: We found that mFOLFOX6 can be a novel treatment option as the first-line treatment for AGC patients with massive ascites or inadequate oral intake.

Keywords: advanced gastric cancer, FOLFOX, massive ascites, inadequate oral intake

\section{Introduction}

Gastric cancer is the fifth most prevalent cancer and the third leading cause of cancer death worldwide, ${ }^{1}$ with $\sim 50,000$ people dying from the malignancy annually. In Japan, it is the second leading cause of cancer death, ${ }^{2}$ and although its mortality rate has decreased over the recent decades, its prevalence has been increasing for both men and women. ${ }^{3}$

Peritoneal metastasis is an important factor to be considered in developing the treatment plan for advanced gastric cancer (AGC). AGC patients are more likely to develop peritoneal metastasis than those with other gastrointestinal cancers, and it is difficult to use an oral medicine due to massive ascites or severe peritoneal metastasis. Furthermore, peritoneal metastasis is among the poor prognostic factors for AGC.,5 
Doublet combinations of platinum and fluoropyrimidines are recommended as a first-line chemotherapy for AGC. In the USA and Europe, combination therapy of platinum and capecitabine is among the preferred regimens. ${ }^{6,7}$ Meanwhile, in Japan, particularly for human epidermal growth factor receptor type 2 (HER2)-negative AGC, combination therapy of platinum and $\mathrm{S}-1$, which is another oral fluoropyrimidine, is used according to the results of the Japan Clinical Oncology Study Group (JCOG) trial (JCOG9912), SPIRITS trial, and G-SOX trial. ${ }^{8-10}$ However, S-1 and cisplatin are difficult to administer in patients with a gastric outlet obstruction or renal dysfunction due to peritoneal metastasis or massive ascites. In general, when we treat AGC patients with inadequate oral intake due to massive ascites or peritoneal metastases, 5-fluorouracil (5-FU)/1-leucovorin (1-LV) is the most widely used based on the result of the JCOG0106 trial. ${ }^{11}$ However, the treatment outcome of 5-FU/1-LV therapy is rather poor compared with the combination therapy of fluoropyrimidines plus platinum.

Oxaliplatin (L-OHP) is a third-generation, platinumbased compound that is active against AGC and has a favorable toxicity profile compared with cisplatin, particularly in patients with renal dysfunction. L-OHP was approved for AGC according to the results of the G-SOX trial in 2014, and in February 2017, the modified FOLFOX6 regimen (mFOLFOX6: a combination of 1-LV and FU with L-OHP) was approved for the treatment of AGC in Japan. ${ }^{12}$ Oh et al reported a Phase II trial of mFOLFOX4 for AGC with ascites, in which a decrease or disappearance of ascites was observed in $35.4 \%$ of patients. ${ }^{13}$ From these results, fluoropyrimidines plus L-OHP may be a promising regimen for patients with severe peritoneal dissemination or massive ascites with inadequate oral intake. However, there were only few reports about the safety and treatment outcomes of mFOLFOX6 therapy for them. This study aimed at evaluating the efficacy and safety of the first-line mFOLFOX6 therapy for AGC patients with massive ascites or inadequate oral intake.

\section{Materials and methods}

\section{Patients}

We retrospectively investigated 364 patients with histopathologically confirmed AGC who were treated with firstline chemotherapy in our institute between November 2015 and May 2018. Patients with peritoneal metastasis and/or those with inadequate oral intake and who received the mFOLFOX6 regimen were included in this study according to the following eligibility criteria: 1) histologically confirmed gastric or gastroesophageal adenocarcinoma; 2) unresectable or recurrent disease; 3) massive ascites and/or inadequate oral intake due to peritoneal metastasis; 4) no previous chemotherapy, except for adjuvant chemotherapy completed more than 6 months before the starting date of mFOLFOX6; 5) adequate bone marrow, hepatic, and renal function; and 6) no previous treatment with L-OHP. This study was performed in accordance with the Declaration of Helsinki and approved by the Institutional Review Board of the Cancer Institute Hospital of the Japanese Foundation for Cancer Research (Registry No 2017-1209). The protocol was described in the Web site of the hospital, and the subjects were provided with the opportunity to opt out, and therefore, no new consent was required from the patients.

\section{Treatments}

The mFOLFOX6 regimen consisted of $85 \mathrm{mg} / \mathrm{m}^{2} \mathrm{~L}-\mathrm{OHP}$, $400 \mathrm{mg} / \mathrm{m}^{2}$ bolus of $5-\mathrm{FU}$, and $400 \mathrm{mg} / \mathrm{m}^{2} 1-\mathrm{LV}$ on the first day, followed by $2,400 \mathrm{mg} / \mathrm{m}^{2}$ of 5 -FU as a continuous infusion (ci) in 46 hours. Treatment was administered every 2 weeks until disease progression, unacceptable toxicity, or patient refusal. Antiemetic prophylaxis with 5-HT3 antagonists and corticosteroids were always administered prior to the L-OHP infusion. If there were adverse reactions of hematological grade 4 or nonhematological grade 3 or 4 , or when the attending physician considered it appropriate, treatment was suspended until recovery, and the dose of 5-FU or L-OHP was reduced.

\section{Definition of ascites and inadequate oral intake}

The levels of ascites were assessed via computed tomography (CT) and categorized as follows: massive, ie, continuous ascites from the pelvic cavity to the upper abdomen; moderate, not massive or mild ascites; and mild, ascites limited to the pelvic cavity or no ascites in middle or upper abdomen. The changes in ascites were also assessed via CT scan and categorized as follows: complete response (CR), disappearance of ascites; partial response (PR), decreased levels of ascites; stable disease (SD), same level of ascites as that before treatment; and progressive disease (PD), increased levels of ascites or drainage frequency. These definitions were the same as those in the multicenter feasibility study of combination therapy with FU, 1-LV, and paclitaxel (PTX) (FLTAX regimen). ${ }^{14}$ Inadequate oral intake was defined as the need for total parenteral nutrition (TPN). This definition was identical to that used in a Phase III trial (JCOG0106) for AGC with peritoneal metastases. ${ }^{11}$ Improvement in oral intake was defined as not needing TPN for more than 7 days. 


\section{Assessments}

Tumor response was assessed via CT imaging and graded using Response Evaluation Criteria in Solid Tumors 1.1. CR was defined as the disappearance of all evidence of disease. PR was defined as a reduction of at least $30.0 \%$ in one-dimensional tumor measurements, without any new lesion or progression of any existing lesion. PD was defined as an increase of at least $20.0 \%$ in the sum of the products of all measurable lesions or the appearance of any new lesion, including the reappearance of any lesion that had disappeared. SD was defined as a tumor response not filling the criteria for $\mathrm{CR}, \mathrm{PR}$, and $\mathrm{PD}$. Toxicity was graded according to the National Cancer Institute Common Terminology Criteria for Adverse Events Version 4.0. Time to treatment failure (TTF) was defined as the duration from the first administration of chemotherapy to the discontinuation of any drugs from any cause. Progression-free survival (PFS) was defined as the duration from the first administration of chemotherapy to the first radiological or clinical observation of disease progression or death from any cause. Overall survival (OS) was defined as the time from the diagnosis of metastatic disease until death. TTF, PFS, and OS were estimated using the Kaplan-Meier method and compared using the log-rank test. All statistical analyses were performed using EZR (Saitama Medical Center, Jichi Medical University, Japan), which is a graphical user interface for R (The R Foundation for Statistical Computing, Vienna, Austria).

\section{Results}

\section{Patient characteristics}

Among the 364 patients with AGC who received first-line therapy, 17 patients ( $4.7 \%$, eleven men and six women) with a median age of 67 (range $=29-74$ ) years were included. The median follow-up time of the study was 8.9 months. A total of four of the 17 patients (23.5\%) had an Eastern Cooperative Oncology Group Performance Status (PS) of 2, and two patients (11.7\%) had HER2-positive tumors. In total, 16 patients had metastasis at the beginning of the treatment, and only one patient had locally advanced tumors. The median number of metastatic organs was 2 (range $=1-5$ ). A total of $13(76.5 \%)$ patients had inadequate oral intake, and four $(23.5 \%)$ had massive ascites (Table 1$)$.

\section{Efficacy}

The median TTF and OS were $4.8(95 \% \mathrm{CI}=1.5-7.5)$ and 8.8 months ( $95 \% \mathrm{CI}=2.3-$ not available), respectively (Figure 1). A total of 10 of the 17 patients $(58.8 \%)$ had target regions, and the objective response rate was $50.0 \%$ (5 of the 10). The median PFS was 4.2 months ( $95 \% \mathrm{CI}=0.46-5.6)$.
Table I Patient demographics and clinical characteristics

\begin{tabular}{|c|c|}
\hline Characteristics & $\begin{array}{l}\text { Total }(\mathrm{N}=17) \\
\text { Number of patients (\%) }\end{array}$ \\
\hline \multicolumn{2}{|c|}{ Age at enrollment, years } \\
\hline Median & 67 \\
\hline Range & $29-74$ \\
\hline \multicolumn{2}{|l|}{ Sex } \\
\hline Male & II (64.7) \\
\hline Female & $6(35.3)$ \\
\hline \multicolumn{2}{|l|}{ ECOG PS } \\
\hline 0 & $2(11.8)$ \\
\hline 1 & II (64.7) \\
\hline 2 & $4(23.5)$ \\
\hline \multicolumn{2}{|c|}{ Macroscopic type } \\
\hline 2 & $3(17.6)$ \\
\hline 3 & $3(17.6)$ \\
\hline 4 & $9(53.0)$ \\
\hline NE & $2(11.8)$ \\
\hline \multicolumn{2}{|l|}{ Histology } \\
\hline Diffuse & $15(88.2)$ \\
\hline Intestinal & $2(11.8)$ \\
\hline \multicolumn{2}{|c|}{ Prior gastrectomy } \\
\hline Yes & $2(11.8)$ \\
\hline No & I5 (88.2) \\
\hline \multicolumn{2}{|c|}{ No metastatic site } \\
\hline I & $5(29.4)$ \\
\hline$\geq 2$ & $12(70.6)$ \\
\hline \multicolumn{2}{|c|}{ Peritoneal metastasis } \\
\hline Yes & $16(94.1)$ \\
\hline No & $\mathrm{I}(5.9)$ \\
\hline \multicolumn{2}{|l|}{ Ascites } \\
\hline Yes & $12(70.6)$ \\
\hline No & $5(29.4)$ \\
\hline Massive & $4(23.5)$ \\
\hline Moderate & $3(17.6)$ \\
\hline Mild & $5(29.4)$ \\
\hline \multicolumn{2}{|c|}{ Inadequate oral intake } \\
\hline Yes & $13(76.5)$ \\
\hline No & $4(23.5)$ \\
\hline
\end{tabular}

Abbreviations: ECOG PS, Eastern Cooperative Oncology Group Performance Status; NE, not evaluated.

Objective improvement in oral intake and ascites was seen in 11 of 13 patients $(84.6 \%)$ and 6 of 12 patients $(50.0 \%$; Table 2). A total of nine patients finished mFOLFOX6 therapy due to the $\mathrm{PD}$, and six of the nine (66.6\%) patients received second-line chemotherapy after the termination of the first-line chemotherapy. The other three patients did not receive any other chemotherapy and were treated with best supportive care. Of the six patients undergoing additional 

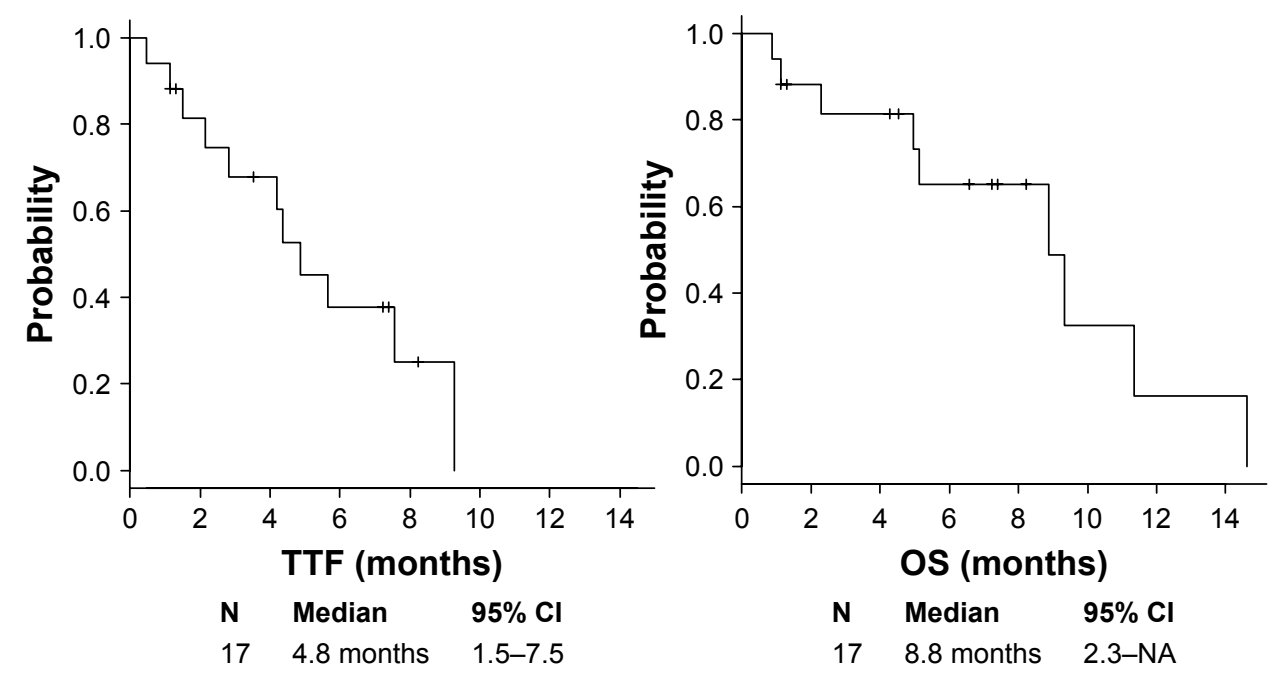

Figure I TTF and OS rate of the study cohort.

Abbreviations: OS, overall survival; TTF, time to treatment failure.

chemotherapeutic regimens, five $(83.3 \%)$ received taxanebased therapy and one (16.7\%) received irinotecan-based therapy. After receiving a second-line chemotherapy with a nontaxane regimen, one patient underwent a taxanecontaining regimen as a third-line chemotherapy. In total, six $(66.6 \%)$ of the nine patients received taxane-based chemotherapy as a second- or third-line chemotherapy.

\section{Adverse events and dose modification}

The major grade 3 or 4 adverse events were neutropenia (35.3\%), febrile neutropenia (5.9\%), fatigue (5.9\%), anorexia

Table 2 Efficacy of mFOLFOX 6 for target regions, ascites, and oral intake

\begin{tabular}{|l|l|}
\hline Target region & $\begin{array}{l}\text { Total }(\mathbf{N}=\text { I 7) } \\
\text { Number of patients (\%) }\end{array}$ \\
\hline Yes & $10(58.8)$ \\
\hline No & $7(4 I .2)$ \\
\hline Objective response rate & $\begin{array}{l}\text { Total }(\mathbf{N}=10) \\
\text { Number of patients (\%) }\end{array}$ \\
\hline Partial response & $5(50.0)$ \\
\hline Stable disease & I (I0.0) \\
\hline Progressive disease & $3(30.0)$ \\
\hline Not evaluated & I (I0.0) \\
\hline Improvement in ascites & $\begin{array}{l}\text { Total }(\mathbf{N}=\text { I 2) } \\
\text { Number of patients (\%) }\end{array}$ \\
\hline Yes & $6(50.0)$ \\
\hline No & $6(50.0)$ \\
\hline Improvement in oral intake & $\begin{array}{l}\text { Total (N=I3) } \\
\text { Number of patients, (\%) }\end{array}$ \\
\hline Yes & II (83.0) \\
\hline No & $2(17.0)$ \\
\hline
\end{tabular}

Abbreviations: FOLFOX, a combination of I-leucovorin and fluorouracil with oxaliplatin.
(5.9\%), and infection (5.9\%); no treatment-related deaths occurred (Table 3). The median relative dose intensity was $90 \%\left(76.5 \mathrm{mg} / \mathrm{m}^{2} / 2\right.$ weeks $)$ for L-OHP, $63.4 \%(253.8 \mathrm{mg} /$ $\mathrm{m}^{2} / 2$ weeks) for 5 -FU bolus infusion, and $99.7 \%(2392.3 \mathrm{mg} /$ $\mathrm{m}^{2} / 2$ weeks) for 5 -FU ci. Dose modification was required in eight (47.0\%) patients due to adverse events (L-OHP: $n=6$; 5-FU bolus infusion: $n=6$; 5 -FU ci: $n=1$ ).

\section{Discussion}

We evaluated the efficacy and safety of the first-line mFOLFOX6 therapy for AGC patients with massive ascites or inadequate oral intake. In this study, ORR was equivalent to the previous first-line chemotherapy without massive ascites or inadequate oral intake,, 10 and the oral intake improvement rate was high although these patients are

Table 3 Adverse events

\begin{tabular}{|l|l|l|l|l|}
\hline Adverse events & Grade 2 & Grade 3 & Grade 4 & $\begin{array}{l}\text { Grade 3-4, } \\
\text { n (\%) }\end{array}$ \\
\hline Hematological \\
\hline Neutropenia & 2 & 4 & 2 & $6(35.3)$ \\
\hline Febrile neutropenia & 0 & $\mathrm{I}$ & 0 & $\mathrm{I}(5.9)$ \\
\hline Thrombocytopenia & $\mathrm{I}$ & 0 & 0 & 0 \\
\hline Nonhematological & 2 & 0 & 0 & 0 \\
\hline Nausea & 2 & 0 & 0 & 0 \\
\hline Constipation & $\mathrm{I}$ & $\mathrm{I}$ & 0 & $\mathrm{I}(5.9)$ \\
\hline Fatigue & 2 & $\mathrm{I}$ & 0 & $\mathrm{I}(5.9)$ \\
\hline Anorexia & 3 & 0 & 0 & 0 \\
\hline $\begin{array}{l}\text { Peripheral } \\
\text { neuropathy }\end{array}$ & $\mathrm{I}$ & & & \\
\hline Infection & 0 & $\mathrm{I}$ & 0 & $\mathrm{I}(5.9)$ \\
\hline Mucositis oral & $\mathrm{I}$ & 0 & 0 & 0 \\
\hline
\end{tabular}


usually not included in clinical trials; there are few reports on the appropriate treatment strategy.

In Japan, although the standard treatment of AGC with inadequate oral intake is 5-FU ci therapy based on the JCOG0106 trial, patients with massive ascites were excluded in that study. In the retrospective analysis of 5-FU ci therapy for massive ascites or inadequate oral intake, the median PFS and OS were 2.4 and 6.0 months, respectively. ${ }^{15}$ Meanwhile, Oh et al conducted a prospective Phase II study to evaluate mFOLFOX4 for AGC patients with malignant ascites $(n=48) .{ }^{13}$ The median PFS and OS were 3.5 and 8.4 months, respectively. All drugs except for 1-LV were administered at a regular dose, and mFOLFOX4 was feasible with grade 3 or 4 neutropenia rates of $19.0 \%$ per cycle, febrile neutropenia rates of $3.0 \%$ per cycle, and nausea and vomiting rates of $6.0 \%$ per person. ${ }^{13}$ However, the treatment schedule of FOLFOX 4 regimen is so complicated, and thus, the mFOLFOX6 regimen is widely used in clinical practice. Recently, Masuishi et al conducted a retrospective study to evaluate mFOLFOX6 for 10 AGC patients with severe peritoneal metastasis. ${ }^{16}$ The median PFS and OS were 7.5 and 13.2 months, respectively. This study included $46.0 \%$ patients with a PS of 2 . The initial dose was modified in $50.0 \%$ of the patients. In addition, the dose was reduced or

Table 4 FOLFOX and FLTAX therapies for AGC with ascites or inadequate oral intake in a literature review of previous reports

\begin{tabular}{|c|c|c|c|c|}
\hline \multirow{2}{*}{$\begin{array}{l}\text { Clinical parameters and } \\
\text { clinical outcomes }\end{array}$} & Oh et $\mathrm{al}^{13}$ & Masuishi et al ${ }^{16}$ & Osumi et al & Iwasa et al ${ }^{14}$ \\
\hline & $\begin{array}{l}\text { Total }(\mathrm{N}=48) \\
\text { Number of patients }(\%)\end{array}$ & $\begin{array}{l}\text { Total }(\mathrm{N}=10) \\
\text { Number of patients }(\%)\end{array}$ & $\begin{array}{l}\text { Total }(\mathrm{N}=17) \\
\text { Number of patients (\%) }\end{array}$ & $\begin{array}{l}\text { Total }(\mathbf{N}=25) \\
\text { Number of patients (\%) }\end{array}$ \\
\hline Chemotherapy & mFOLFOX4 & mFOLFOX6 & mFOLFOX6 & FLTAX \\
\hline \multicolumn{5}{|l|}{ Age (years) } \\
\hline Median & 60 & 64.5 & 67 & 65 \\
\hline Range & $60-70$ & $40-94$ & $29-74$ & $55-75$ \\
\hline \multicolumn{5}{|l|}{ Gender } \\
\hline Female & $16(33.3)$ & $8(80.0)$ & II (64.7) & $15(60.0)$ \\
\hline Male & $32(66.7)$ & $2(20.0)$ & $6(35.3)$ & $10(40.0)$ \\
\hline \multicolumn{5}{|l|}{ ECOG PS } \\
\hline $0-1$ & $26(54.2)$ & $5(50.0)$ & $13(76.5)$ & $20(80.0)$ \\
\hline$\geq 2$ & $22(45.8)$ & $5(50.0)$ & $4(23.5)$ & $5(20.0)$ \\
\hline \multicolumn{5}{|l|}{ No metastatic site } \\
\hline I & $30(62.5)$ & $5(50.0)$ & $5(29.4)$ & NE \\
\hline$\geq 2$ & $18(37.5)$ & $5(50.0)$ & $12(70.6)$ & NE \\
\hline \multicolumn{5}{|l|}{$\begin{array}{l}\text { Prior palliative } \\
\text { chemotherapy }\end{array}$} \\
\hline 0 & $21(43.8)$ & $10(100.0)$ & $17(100.0)$ & I8 (72.0) \\
\hline$\geq 1$ & $27(56.2)$ & $0(0.0)$ & $0(0.0)$ & $7(28.0)$ \\
\hline \multicolumn{5}{|l|}{ Measurable lesion } \\
\hline Yes & $30(62.5)$ & $3(30.0)$ & $10(58.8)$ & NE \\
\hline No & $18(37.5)$ & $7(70.0)$ & $7(4 \mid .2)$ & NE \\
\hline Objective response rate & $12(33.3)$ & $3(100.0)$ & $5(50.0)$ & NE \\
\hline \multicolumn{5}{|l|}{ Ascites } \\
\hline Yes & $48(100.0)$ & $9(90.0)$ & $12(70.6)$ & $24(96.0)$ \\
\hline No & $0(0.0)$ & $I(I 0.0)$ & $5(29.4)$ & I (4.0) \\
\hline Improvement of ascites & $17(35.4)$ & $7(78.0)$ & $6(50.0)$ & II (45.8) \\
\hline \multicolumn{5}{|l|}{ Inadequate oral intake } \\
\hline Yes & NE & $7(70.0)$ & $13(76.4)$ & $12(48.0)$ \\
\hline No & NE & $3(30.0)$ & $4(23.6)$ & $13(52.0)$ \\
\hline $\begin{array}{l}\text { Improvement in oral } \\
\text { intake }\end{array}$ & NE & $4(57.0)$ & II (83.0) & NE \\
\hline PFS (median, months) & 3.5 & 7.5 & 4.2 & 6.2 \\
\hline OS (median, months) & 8.4 & 13.2 & 8.8 & 9.5 \\
\hline
\end{tabular}

Abbreviations: AGC, advanced gastric cancer; ECOG PS, Eastern Cooperative Oncology Group Performance Status; FLTAX, a combination of I-leucovorin and fluorouracil with paclitaxel; FOLFOX, a combination of l-leucovorin and fluorouracil with oxaliplatin; NE, not evaluated; OS, overall survival; PFS, progression-free survival. 
interrupted in the other five patients because of adverse events of mFOLFOX6. In this study, the PFS and OS were 4.2 and 8.8 months, respectively. Eight (47.0\%) patients needed dose modification because of adverse events of mFOLFOX6, mainly neutropenia (Table 4). Notably, the rate of objective improvement in oral intake was higher than that in previous reports $(11$ of $13,84.6 \%) .{ }^{15}$ In the study by Hara et al in AGC patients with peritoneal metastasis who were treated with 5-FU/LV, only $17(33.0 \%)$ patients improved to the point of needing no TPN. ${ }^{15}$ These data suggest that doublet combinations of platinum and fluoropyrimidines are more effective than 5-FU ci therapy even for the AGC patients with massive ascites or inadequate oral intake.

Another promising regimen for AGC with peritoneal metastasis is FLTAX and hyperthermic intraperitoneal chemotherapy (HIPEC). In the Phase I/II study of FLTAX for the same population, the median PFS and OS were 6.2 and 9.5 months, respectively (Table 4). ${ }^{14}$ PTX is usually used as a standard second-line treatment, ${ }^{17}$ and based on the results of these previous studies, the Phase III JCOG1108/ WJOG7312G trial comparing FLTAX with 5-FU/LV for the same population is ongoing in Japan. Meanwhile, for patients with advanced peritoneal metastases, the findings of randomized trials in Asia support the use of cytoreductive surgery plus HIPEC in selected patients. ${ }^{18}$ However, randomized data for non-Asian patients are lacking. Currently, this approach cannot be recommended outside the context of clinical research. Further studies are warranted.

There were some limitations in this study. This was a retrospective study, and the sample size was also small because most patients had poor PS and are thus ineligible for chemotherapy. Although treatment outcomes in this study are insufficient compared with previous studies on other first-line treatment regimens such as S-1 plus L-OHP or S-1 plus cisplatin, mFOLFOX6 may be among the treatment options for AGC patients with massive ascites or inadequate oral intake who do not fulfill eligibility criteria of chemotherapy using oral fluoropyrimidines.

\section{Conclusion}

Our findings indicate that mFOLFOX6 yields clinical benefit. mFOLFOX6 therapy is feasible and effective and could be among the treatment options for first-line chemotherapy for AGC patients with massive ascites or inadequate oral intake.

\section{Acknowledgments}

We thank the data manager Yuki Horiike. The abstract of this paper was presented at the ESMO 20th World Congress on Gastrointestinal Cancer as a poster presentation. The poster's abstract was published in "Poster Abstracts" in Annals of Oncology, Volume 29, Issue suppl_5, 1 June 2018: https://academic.oup.com/annonc/article/29/suppl 5/ mdy151.083/5039400.

\section{Disclosure}

The authors report no conflicts of interest in this work.

\section{References}

1. Japan Ministry of Health, Labour and Welfare. Vital Statistics of Japan Ministry of Health, Labour and Welfare. Available from: http://www. mhlw.go.jp/toukei/list/81-1.html. Accessed August 27, 2018.

2. Center for Cancer Control and Information Services. Available from: http://ganjoho.jp/reg_stat/index.html. Accessed August 27, 2018.

3. GLOBOCAN 2012. Estimated cancer incidence, mortality and prevalence worldwide; 2012. Available from: http://globocan.iarc.fr/. Accessed April 2, 2018.

4. Chau I, Norman AR, Cunningham D, Waters JS, Oates J, Ross PJ. Multivariate prognostic factor analysis in locally advanced and metastatic esophago-gastric cancer - pooled analysis from three multicenter, randomized, controlled trials using individual patient data. J Clin Oncol. 2004;22(12):2395-2403.

5. Koo DH, Ryoo BY, Kim HJ, et al. A prognostic model in patients who receive chemotherapy for metastatic or recurrent gastric cancer: validation and comparison with previous models. Cancer Chemother Pharmacol. 2011;68(4):913-921.

6. Ajani JA, D'Amico TA, Almhanna K, et al. Gastric cancer, version 3.2016, NCCN clinical practice guidelines in oncology. J Natl Compr Canc Netw. 2016;14(10):1286-1312.

7. Smyth EC, Verheij M, Allum W, et al. Gastric cancer: ESMO clinical practice guidelines for diagnosis, treatment and follow-up. Ann Oncol. 2016;27(Suppl 5):v38-v49.

8. Boku N, Yamamoto S, Fukuda H, et al. Fluorouracil versus combination of irinotecan plus cisplatin versus S-1 in metastatic gastric cancer: a randomised phase 3 study. Lancet Oncol. 2009;10(11):1063-1069.

9. Koizumi W, Narahara H, Hara T, et al. S-1 plus cisplatin versus S-1 alone for first-line treatment of advanced gastric cancer (SPIRITS trial): a phase III trial. Lancet Oncol. 2008;9(3):215-221.

10. Yamada Y, Higuchi K, Nishikawa K, et al. Phase III study comparing oxaliplatin plus S-1 with cisplatin plus S-1 in chemotherapynaïve patients with advanced gastric cancer. Ann Oncol. 2015;26(1): 141-148.

11. Shirao K, Boku N, Yamada Y, et al. Randomized Phase III study of 5-fluorouracil continuous infusion vs. sequential methotrexate and 5 -fluorouracil therapy in far advanced gastric cancer with peritoneal metastasis (JCOG0106). Jpn J Clin Oncol. 2013;43(10):972-980.

12. Japanese Gastric Cancer Association. Japanese gastric cancer treatment guidelines 2018 (ver. 5) [in Japanese]. 2018:1-91.

13. Oh SY, Kwon HC, Lee S, et al. A Phase II study of oxaliplatin with low-dose leucovorin and bolus and continuous infusion 5-fluorouracil (modified FOLFOX-4) for gastric cancer patients with malignant ascites. Jpn J Clin Oncol. 2007;37(12):930-935.

14. Iwasa S, Goto M, Yasui H, et al. Multicenter feasibility study of combination therapy with fluorouracil, leucovorin and paclitaxel (FLTAX) for peritoneal disseminated gastric cancer with massive ascites or inadequate oral intake. Jpn J Clin Oncol. 2012;42(9):787-793.

15. Hara H, Kadowaki S, Asayama M, et al. First-line bolus 5-fluorouracil plus leucovorin for peritoneally disseminated gastric cancer with massive ascites or inadequate oral intake. Int J Clin Oncol. 2018;23(2): 275-280.

16. Masuishi T, Kadowaki S, Kondo M, et al. FOLFOX as first-line therapy for gastric cancer with severe peritoneal metastasis. Anticancer Res. 2017;37(12):7037-7042. 
17. Hironaka S, Ueda S, Yasui H, et al. Randomized, open-label, phase III study comparing irinotecan with paclitaxel in patients with advanced gastric cancer without severe peritoneal metastasis after failure of prior combination chemotherapy using fluoropyrimidine plus platinum: WJOG 4007 trial. J Clin Oncol. 2013;31(35):4438-4444.
18. Yang XJ, Huang CQ, Suo T, et al. Cytoreductive surgery and hyperthermic intraperitoneal chemotherapy improves survival of patients with peritoneal carcinomatosis from gastric cancer: final results of a phase III randomized clinical trial. Ann Surg Oncol. 2011;18(6):1575-1581.

\section{Publish your work in this journal}

OncoTargets and Therapy is an international, peer-reviewed, open access journal focusing on the pathological basis of all cancers, potential targets for therapy and treatment protocols employed to improve the management of cancer patients. The journal also focuses on the impact of management programs and new therapeutic agents and protocols on

\section{Dovepress}

patient perspectives such as quality of life, adherence and satisfaction. The manuscript management system is completely online and includes a very quick and fair peer-review system, which is all easy to use. Visit http://www.dovepress.com/testimonials.php to read real quotes from published authors.

Submit your manuscript here: http://www.dovepress.com/oncotargets-and-therapy-journal 